\title{
May Dental Extraction Trigger Subacute Thyroiditis?
}

\author{
Dr. Kevser Onbasıl ${ }^{1}$, Dr. Hasan Hatipoğllu², Dr. Serdar Ü̈gü̈n ${ }^{3}$, Dr. Müjgan Güngör Hatipoğllu
}

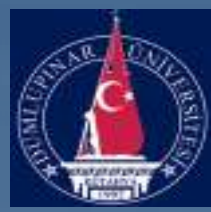

Faculty of Medicine, Department of Endocrinology ${ }^{1}$, Faculty of Dentistry, Department of

Periodontology 2, Faculty of Medicine, Department of Internal Medicine ${ }^{3}$ and Faculty of Dentistry

Department of Radiology 4, Dumlupınar University, Kütahya, Turkey.

\begin{abstract}
Introduction: Subacute thyroiditis (SAT) is a self-limiting thyroid disease associated with a triphasic clinical course of hyperthyroidism, hypothyroidism, and return to normal thyroid function. The well-known clinical features of SAT include thyroid pain with symptoms of hyperthyroidism, suppressed level of thyroid-stimulating hormone (TSH), low thyroid uptake of radioactive iodine, and elevated erythrocyte sedimentation rate. Diagnosis is based on clinical and laboratory data, and tissue diagnosis is rarely required. Here we report about two cases that developed SAT after dental extraction. Most cases of SAT develop after systemic viral infections like Coxsackie, cytomegalovirus, Epstein-Barr virus, and adenovirus. SAT (de Quervain's) presents typically with neck pain radiating to the ear. To our knowledge there has been no report about SAT developing after dental extraction. The possible complications of tooth extraction is blood loss, impairment of labial sensation, oro-antral fistula, fracture of the abscesses, endocarditis and bacteremia, but SAT has not been reported.

Cases: Our first case was a 45-year-old woman who experienced fever and neck pain after three days of dental extraction. Her sedimentation rate was elevated and hyperthyroidism was detected. She was started steroid treatment with the diagnosis of SAT. After 1 week her symptoms resolved and her sedimentation rate and suppressed TSH levels returned to normal after 1 month. The second patient was a 44-year-old woman who underwent a dental extraction and experienced neck pain after 4-5 days after the procedure. Her sedimentation rate was elevated and FT4 level was increased and TSH levels was suppressed. Her uptake was low which was performed in another hospital. She was started methyl prednisolone and her symptoms resolved after 1 week and laboratory parameters were normal after 6 weeks.

Conclusion: We have observed two cases experiencing subacute thyroiditis after dental extraction.
\end{abstract}

\section{INTRODUCTION}

Subacute thyroiditis (subacute granulomatous thyroiditis) (SAT) is characterized by neck pain or discomfort and a tender diffuse goiter. It is a self-limiting thyroid disease. Usually it begins with hyperthyroidism and is followed by euthyroidism, hypothyroidism, and ultimately restoration of normal thyroid function (1). The well-known clinical features of SAT include thyroid pain with symptoms of hyperthyroidism, suppressed level of thyroid-stimulating hormone (TSH), low thyroid uptake of radioactive iodine, and elevated erythrocyte sedimentation rate. Diagnosis is based on clinical and laboratory data and tissue diagnosis is rarely required (2). Here we report about two cases that developed SAT after dental extraction. Most cases of SAT develop after systemic viral infections or a postviral inflammatory process triggers it. SAT presents typically with neck pain and may radiate to the upper neck, jaw, throat, upper chest, or ears. Fever, fatigue, malaise, anorexia, and myalgia are common accompanying symptoms (1). To our knowledge there has been no report about SAT developing after dental extraction.

Some of the possible complications of tooth extraction/s are pain, excessive and prolonged bleeding, postoperative infection-swelling, maxillary sinus perforations, nerve injury (inferior alveoler, mental and lingual nerve), accidentally displacement of tooth or root pieces into anatomical spaces, fractures of bone structures, trismus etc. $(3,4)$. On other hand different dental procedures are able to produce substantially bacteremia (5). To our knowledge there has been no report about SAT developing after dental extraction

\section{CASES}

Case 1: Our first case was a 45 year old woman who experienced fever and neck pain after three days of dental extraction. On physical examination she had palpable thyroid which was consistent with grade 2 enlargement and elevated body temperature $(38,50 \mathrm{C})$. erythrocyte sedimentation rate was elevated $(80 \mathrm{~mm} / \mathrm{h})$ (normal range, $0-20 \mathrm{~mm} / \mathrm{h}$ ); and hyperthyroidism was detected (TSH 0,06 $\mu \mathrm{U} / \mathrm{ml}$ (normal range 0,3-4), and FrT4 was $1.76 \mathrm{ng} / \mathrm{ml}(\mathrm{N}: 1.0-1.6)$. CRP level was $9.17 \mathrm{mg} / \mathrm{dl}$ (normal range, $0.00-0.50 \mathrm{mg} / \mathrm{dl}$ ) elevated. Thyroid scintigraphy revealed a low thyroid Tc-99m pertechnetate uptake. Anti-TPO antibody and anti-Tg antibody was negative. Biochemistry, complete blood count, and liver and kidney function tests were normal. She was started steroid treatment (methylprednisolone
$40 \mathrm{mg} /$ day) with the diagnosis of SAT. The dose was tapered weekly and was stopped after six weeks. After one week her symptoms resolved and her sedimentation rate and suppressed TSH levels returned to normal after one month.

Case 2: The second patient was a 44 year old woman who underwent a dental extraction and experienced neck pain after 4 to 5 days of the procedure. She was attending an otolaryngology outpatient clinic and received sulbactam-ampicillin treatment with the diagnosis of upper respiratory tract infection. Her fever persisted $(37,8 \mathrm{OC})$ and neck pain did not resolve. She experienced palpitations and insomnia for the last week. Her pulse rate was 96 beats/min and thyroid was minimally palpable. Her erythrocyte sedimentation rate was elevated (80 $\mathrm{mm} / \mathrm{h})($ normal range, $0-20 \mathrm{~mm} / \mathrm{h})$ and FT4 level $2.67 \mathrm{ng} / \mathrm{ml}(\mathrm{N}: 1.0-$ 1.6) was increased and TSH level (TSH: $0,01 \mu \mathrm{U} / \mathrm{ml}$ (normal range $0,3-$ 4) was suppressed. Her thyroid scintigraphy uptake was low which had been performed in another hospital and she was referred to our endocrinology outpatient clinic. With all these findings the patient was diagnosed with SAT. After carefully taken history she told us her dental extraction history. She was started niethylprednisolone (32mg/day and the dose was tapered weekly and stopped after six weeks) and her symptoms resolved after one week and laboratory parameters returned to normal after six weeks.

\section{DISCUSSION}

Subacute thyroiditis may follow systemic viral infection like influenza, Coxsackie virus, cytomegalovirus, Epstein-Barr virus and adenovirus. It is a rare entity and may remain undiagnosed if unusual systemic manifestations are present. Subacute thyroiditis following viral infection may present as fever of unknown origin, and if the clinician does not consider the other accompanying reasons it can be overlooked. Accompanying jaw and ear pain should prompt the clinician to suspect of the diagnosis of the SAT (5). Viral-induced subacute thyroiditis (de Quervain's) due to various viruses rarely has been reported so far (6), but SAT following dental extraction has not been reported so far.

\section{CONCLUSION}

We have observed to cases experiencing subacute thyroiditis after dental extraction. To the best of our knowledge, these are the first cases of de Quervain's subacute thyroiditis in which dental extraction may have been triggered the process
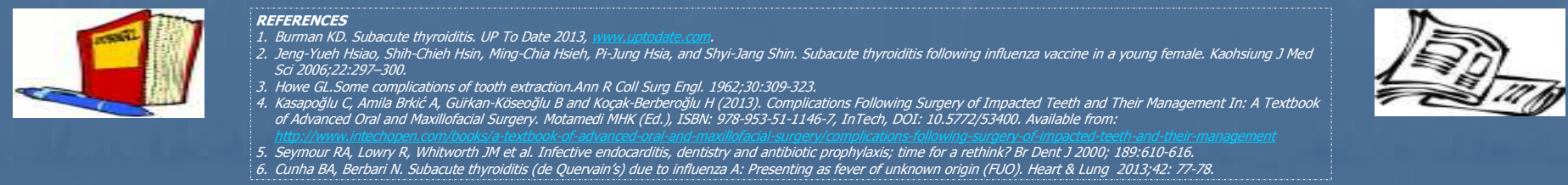